Artigo / Article

\title{
Avaliação da incidência das mutações G1691A no gene do fator $V$ (fator V Leiden) e G20210A no gene da protrombina em pacientes com diabetes mellitus tipo 2 \\ Incidence of G1691A factor $V$ gene (factor V Leiden) and G20210A prothrombin gene mutations in type 2 diabetes patients
}

\author{
Anna L. Soares ${ }^{1}$ \\ Marinez O. Sousa ${ }^{2}$ \\ Marcelo C. Lasmar ${ }^{3}$ \\ Micheline L. Garcia \\ Bethânia A. Novelli ${ }^{4}$ \\ Geralda F. G. Lages ${ }^{5}$ \\ Jarbas E. Cardoso \\ Luci M. S. Dusse ${ }^{2}$ \\ Lauro M. Vieira ${ }^{2}$ \\ Ana P. Fernandes ${ }^{2}$ \\ Maria G. Carvalho ${ }^{2}$
}

\begin{abstract}
Em virtude da alta prevalência de Diabetes mellitus tipo 2 (DM2) na população mundial e da alta taxa de mortalidade decorrente de eventos trombóticos, é de extrema importância o conhecimento das alterações no sistema hemostático em pacientes portadores deste distúrbio. A mutação no gene do fator V (G1691A - fator V Leiden) em heterozigose ou homozigose confere aos portadores ofenótipo de resistência à proteina $C$ ativada, situação que aumenta em sete vezes o risco de desenvolver uma trombose. A mutação G20210A no gene da protrombina resulta no quadro de hiperprotrombinemia, aumentando o risco de trombose em três vezes. A pesquisa dessas mutações de interesse em trombofilia é de grande relevância considerando que a presença das mesmas pode exacerbar o estado de hipercoagulabilidade acelerando as complicações no diabetes. O presente estudo teve como objetivo avaliar a incidência dessas mutações em individuos hígidos (Controle, $n=16)$, pacientes com DM2 $(n=7)$, com hipertensão (HAS, $n=12)$ e com DM2+HAS (n=18), através da técnica de PCR-RFLP. As freqüencias encontradas nos grupos estudados foram baixas e similares àquelas observadas na população brasileira em geral. Não foi possivel estabelecer correlação entre a presença da mutação e características específicas de cada grupo. Dessa forma, ainda não está claro se há ou não uma maior prevalência dessas mutações em individuos diabéticos e se a presença das mesmas contribui para o aumento do risco de desenvolver trombose nesses individuos, sendo necessário estudos mais amplos para a elucidação da questão. Rev. bras. hematol. hemoter. 2005;27(3):192-196.
\end{abstract}

Palavras-chave: Diabetes tipo 2; fator V Leiden; mutação no gene da protrombina.

\section{Introdução}

Em 1963, Egeberg demonstrou pela primeira vez o aumento da coagulação sangüínea em pacientes com DM, e em 1966, Davis et al encontraram depósitos de fibrina em lesão glomerular diabética. Desde então, numerosos estudos sobre coagulação em pacientes diabéticos têm sido conduzidos. ${ }^{1}$
Dados de 2000 indicam que $80 \%$ dos pacientes com DM morrem por trombose e $75 \%$ dessas mortes resultam de eventos cardiovasculares. Anormalidades endoteliais, indubitavelmente, apresentam um papel importante no aumento da ativação de plaquetas e dos fatores da coagulação em diabéticos. $^{2}$

O DM2 é a forma mais freqüente e corresponde a cerca de $90 \%$ dos casos de DM no mundo. ${ }^{3}$ Estima-se que $70 \%$

\footnotetext{
${ }^{I}$ Farmacêutica, doutoranda em Ciências Farmacêuticas - UFMG.

${ }_{3}^{2}$ Professores adjuntos do Departamento de Análises Clínicas e Toxicológicas da Faculdade de Farmácia da UFMG.

Bolsistas de Iniciação Científica - CNPq.

${ }_{5}^{4}$ Médica da Santa Casa de Misericórdia de Belo Horizonte.

Farmacêuticos bioquímicos do Departamento de Análises Clínicas e Toxicológicas da Faculdade de Farmácia da UFMG.
} 
dos indivíduos com DM2 apresentam hipertensão arterial sistêmica (HAS) concomitantemente, ${ }^{4}$ condição que também promove disfunção endotelial, comprometendo a hemostasia.

A complicação vascular diabética é obviamente de etiologia multifatorial. O DM está associado a uma variedade de complicações de pequenos e grandes vasos como retinopatia, nefropatia, neuropatia, IAM, derrame e doenças em vasos periféricos, que são freqüentemente responsáveis pela morte prematura dos pacientes.

Eventos macrovasculares são mais freqüentemente relacionados com processos trombóticos de artérias de grande calibre, enquanto a microangiopatia parece ser o resultado de um desarranjo funcional e metabólico nos pequenos vasos. ${ }^{1}$ Entretanto, essas alterações vasculares não explicam todos os eventos trombóticos em pacientes com DM2.

Bertina et $a l^{5}$ demonstraram que o fenótipo de resistência à ação da proteína $\mathrm{C}$ ativada $(\mathrm{PCa})$ estava associado à heterozigose ou homozigose para uma mutação pontual no gene do fator V (fator V Leiden). Tal mutação consiste de uma substituição de uma guanina $(\mathrm{G})$ por uma adenina (A) na posição 1691, o que acarreta a troca de uma arginina por uma glicina na posição 506 da proteína.

A PCa é uma serinoprotease que, ligada ao seu cofator PS, tem a capacidade de limitar a formação do coágulo pela inativação dos co-fatores Va e VIIIa. O fator Va Leiden não é susceptível à proteólise, permanecendo, assim, disponível para a formação do complexo protrombinase da cascata da coagulação, favorecendo a formação de trombina, ou seja, contribuindo para o estado de hipercoagulabilidade. ${ }^{6}$ Indivíduos portadores dessa mutação têm um risco sete vezes maior de desenvolver um trombo do que os não portadores. ${ }^{5}$

Poort et al ${ }^{7}$ identificaram a mutação G20210A no gene da protrombina. Essa mutação é responsável pelo aumento da concentração plasmática de protrombina, aumentando o risco de trombose em três vezes.

A mutação não altera a estrutura da proteína, mas induz uma elevação dos níveis plasmáticos de protrombina e o resultado final é um estado potencial de hipercoagulabilidade. ${ }^{6} \mathrm{O}$ mecanismo pelo qual a mutação promove o aumento dos níveis de protrombina ainda não foi completamente elucidado. Como a mutação G20210A localiza-se na extremidade 3' do gene, pode ser que se tenha uma eficiência maior na transcrição ou uma maior estabilidade do mRNA transcrito. ${ }^{7}$

Diante da importância de tais mutações no aumento do risco de desenvolver trombose e pelo fato de que o diabetes também favorece a formação de trombos, o presente estudo teve como objetivo avaliar a incidência dessas mutações em indivíduos hígidos (Controle, $\mathrm{n}=16$ ), pacientes com DM2 $(\mathrm{n}=7)$, com hipertensão (HAS, $\mathrm{n}=12$ ) e com DM2+HAS ( $\mathrm{n}=18$ ), todos sem lesão vascular evidente (todos participantes eram normoalbuminúricos).

\section{Casuística e Métodos}

O presente trabalho foi aprovado pelo Comitê de Ética em Pesquisa (CEP) da Santa Casa, protocolado sob o número 021/2003 e pelo Comitê de Ética em Pesquisa da UFMG (Coep) sob o número 074/03. Os 53 indivíduos participantes deste estudo compuseram quatro distintos grupos:

1) Grupo-controle (Controle): constituído de 16 indivíduos hígidos, sob o ponto de vista clínico e laboratorial.

2) Grupo com hipertensão (HAS): constituído de 12 indivíduos normoalbuminúricos com diagnóstico prévio de hipertensão e que não apresentavam, no momento da coleta, outras manifestações clínicas e laboratoriais.

3) Grupo com DM2 (DM2): constituído de sete indivíduos normoalbuminúricos com diagnóstico prévio de DM2 e que não apresentavam, no momento da coleta, outras manifestações clínicas e laboratoriais.

4) Grupo com DM2 e hipertensão (DM2+HAS): constituído de 18 indivíduos normoalbuminúricos com diagnóstico prévio de DM2 e hipertensão, e que não apresentavam, no momento da coleta, outras manifestações clínicas e laboratoriais.

Os indivíduos com diagnóstico clínico prévio de DM2, segundo os critérios da $\mathrm{WHO},{ }^{8}$ ou que estivessem fazendo uso de medicamentos antidiabéticos, foram classificados como DM2, e os indivíduos com pressão sangüínea igual ou superior a $140 \mathrm{mmHg}$ para a pressão arterial sistólica ou 90 $\mathrm{mmHg}$ para a pressão arterial diastólica, ou que estivessem fazendo uso de medicação anti-hipertensiva, foram classificados como hipertensos. ${ }^{9}$ Foram excluídos do estudo indivíduos com doença renal crônica - concentração de creatinina sérica igual ou superior a $2,0 \mathrm{mg} / \mathrm{dL}^{10}$; indivíduos com bacteriúria e hematúria, com função anormal do fígado com alanina aminotransferase (ALT), aspartato aminotransferase (AST) ou fosfatase alcalina (FAL) com valores acima de duas vezes o limite superior de referência, ${ }^{11}$ com história clínica de doença cardíaca coronariana, derrame, tumor maligno, doença infecciosa aguda, ou que estavam em uso de medicamento com efeito sob a função hemostática.

As amostras de sangue venoso foram obtidas entre 8:00 e 10:00 h da manhã, após jejum de 12 a 14 horas. Foram realizados exames bioquímicos de rotina para caracterização dos grupos. As amostras de urina foram coletadas em frascos apropriados, após higienização prévia. Foi coletado o jato médio da primeira urina da manhã ou da segunda após três horas de retenção para a realização do exame sumário e microalbuminúria.

A identificação da troca de uma $G$ por uma A na posição 1691 do gene do fator $\mathrm{V}$ foi realizada como descrito previamente por Bertina. ${ }^{5}$

A reação de PCR foi executada utilizando-se os oligonucleotídeos 5'-TCA GGC AGG AAC AAC ACC AT-3' e 5'-GGT TAC TTC AAG GACAAAATA CCT GTAAAG CT-3'. As condições da reação de PCR consistiram em 40 ciclos de 
desnaturação a $94^{\circ} \mathrm{C}$ por um minuto, anelamento a $55^{\circ} \mathrm{C}$ por um minuto e extensão a $72^{\circ} \mathrm{C}$ por um minuto, precedidos de um passo inicial de desnaturação a $94^{\circ} \mathrm{C}$ por dez minutos e finalizados com uma etapa de $72^{\circ} \mathrm{C}$ por cinco minutos.

$\mathrm{O}$ produto de $\mathrm{PCR}$ de $241 \mathrm{pb}$ foi submetido à digestão com a enzima de restrição Hind III (Promega ${ }^{\circledR}$ ). A presença da mutação cria uma seqüência que a enzima Hind III reconhece e leva à quebra do fragmento em duas partes, uma de $209 \mathrm{pb}$ e outra de $32 \mathrm{pb}$. Indivíduos heterozigotos para a mutação apresentam três fragmentos $(241,209$ e 32pb) e indivíduos homozigotos apresentam dois fragmentos (209 e 32pb). $O$ produto da digestão foi analisado por eletroforese em gel de poliacrilamida a $6 \%$, seguido de coloração pela prata.

A identificação da troca de uma $G$ por uma Ana posição 20210 do gene do fator II foi realizada como descrito previamente por Poort.?

A reação de PCR foi executada utilizando-se os oligonucleotídeos 5'-TCT AGAAAC AGT TGC CTG GC-3' e 5'-ATA GCA CTG GGAGCATTGAAGC-3'.As condições da reação de PCR consistiram em 40 ciclos de desnaturação a $94^{\circ} \mathrm{C}$ por um minuto, anelamento a $57^{\circ} \mathrm{C}$ por um minuto e extensão a $72^{\circ} \mathrm{C}$ por um minuto, precedidos de um passo inicial de desnaturação a $94^{\circ} \mathrm{C}$ por dez minutos e finalizados com um passo de $72^{\circ} \mathrm{C}$ por cinco minutos.

O produto de PCR de $345 \mathrm{pb}$ foi submetido à digestão com a enzima de restrição Hind III. A presença da mutação cria uma seqüência que a enzima Hind III reconhece e leva à quebra do fragmento em duas partes, uma de $322 \mathrm{pb}$ e outra de $23 \mathrm{pb}$.

Indivíduos heterozigotos para a mutação apresentam três fragmentos ( 345 , 322 e $23 \mathrm{pb}$ ) e indivíduos homozigotos apresentam dois fragmentos (322 e 23pb). $\mathrm{O}$ produto da digestão foi analisado por eletroforese em gel de poliacrilamida a $8 \%$, seguido de coloração pela prata.
O padrão de peso molecular "1Kb Plus" (Gibco $\left.{ }^{\circledR}\right)$ foi utilizado como referência nas eletroforeses. Os fragmentos com menos de 100 pb não são visualizados no gel corado.

Os resultados dos grupos estão expressos pelos valores das médias e dos desvios padrão. Foi realizado o teste de análise de variância multipareada (Anova), para comparação dos grupos, utilizando-se o programa Sigma Stat ${ }^{\circledR}$ versão 1.0. Valor de $\mathrm{p}$ menor que 0,05 foi considerado estatisticamente significante.

\section{Resultados}

A Tabela 1 apresenta as características clínicas e metabólicas dos grupos Controle, com hipertensão (HAS), com DM2 (DM 2) e com DM2 e hipertensão (DM2+HAS).

Tabela 1

Características clínicas e metabólicas dos grupos estudados

\begin{tabular}{|c|c|c|c|c|c|}
\hline & Controle & HAS & DM2 & DM2+HAS & $\mathrm{p}$ \\
\hline $\mathrm{n}(\mathrm{m} / \mathrm{h})$ & $16(9 / 7)$ & $12(8 / 4)$ & $7(3 / 4)$ & $18(16 / 2)$ & \\
\hline Idade (anos) & $52,3 \pm 5,4$ & $54,8 \pm 5,2$ & $52,1 \pm 8,3$ & $57,2 \pm 6,0$ & \\
\hline IMC (kg/m2) & $25,1 \pm 3,9$ & $28,4 \pm 3,6$ & $28,1 \pm 3,5$ & $28,6 \pm 4,1$ & \\
\hline $\mathrm{GLI}(\mathrm{mg} / \mathrm{dL})$ & $91,7 \pm 9,3$ & $91,0 \pm 9,9$ & $154,6 \pm 55,2^{a, b}$ & $167,6 \pm 57,3^{a, b}$ & $\begin{array}{l}\mathrm{p}^{\mathrm{a}}<0,0001 \\
\mathrm{p}^{\mathrm{b}}<0,0001\end{array}$ \\
\hline GHB (\%) & $6,4 \pm 1,5$ & $7,2 \pm 2,1$ & $8,6 \pm 3,2$ & $8,9 \pm 2,9^{a}$ & $p^{a}=0,02$ \\
\hline GLI PP (mg/dL) & $102,8 \pm 16,6$ & $103,3 \pm 19,8$ & $184,7 \pm 122,1$ & $151,5 \pm 69,2^{a, b}$ & $\begin{array}{l}p^{a}=0,01 \\
p^{b}=0,04\end{array}$ \\
\hline $\mathrm{CT}(\mathrm{mg} / \mathrm{dL})$ & $180,4 \pm 27,9$ & $198,4 \pm 44,5$ & $201,7 \pm 44,2$ & $190,6 \pm 44,5$ & ns \\
\hline $\mathrm{HDLc}(\mathrm{mg} / \mathrm{dL})$ & $47,5 \pm 10,1$ & $40,0 \pm 13,2$ & $41,0 \pm 7,0$ & $39,6 \pm 12,0^{a}$ & $\mathrm{p}^{\mathrm{a}}=0,046$ \\
\hline LDLc (mg/dL) & $116,3 \pm 29,8$ & $129,9 \pm 39,7$ & $137,4 \pm 46,0$ & $117,5 \pm 37,0$ & ns \\
\hline $\mathrm{TGL}(\mathrm{mg} / \mathrm{dL})$ & $82,9 \pm 45,2$ & $142,5 \pm 69,2^{\mathrm{a} 1}$ & $116,7 \pm 53,1$ & $168,0 \pm 75,0$ а2 & $\begin{array}{c}\mathrm{p}^{\mathrm{a} 1}=0,009 \\
\mathrm{p}^{\mathrm{a} 2}=0,0004\end{array}$ \\
\hline $\mathrm{ALT}(\mathrm{U} / \mathrm{L})$ & $22,5 \pm 9,4$ & $18,8 \pm 10,6$ & $22,3 \pm 8,8$ & $23,6 \pm 13,3$ & ns \\
\hline AST (U/L) & $27,8 \pm 8,3$ & $21,4 \pm 4,8$ & $20,3 \pm 6,5$ & $23,5 \pm 13,4$ & ns \\
\hline FAL (U/L) & $29,5 \pm 9,2$ & $38,5 \pm 10,0^{a 1}$ & $39,7 \pm 15,7$ & $40,9 \pm 11,4^{\mathrm{a} 2}$ & $\begin{array}{c}\mathrm{p}^{\mathrm{a} 1}=0,02 \\
\mathrm{p}^{\mathrm{a} 2}=0,003\end{array}$ \\
\hline $\mathrm{Na}+(\mathrm{mEq} / \mathrm{L})$ & $139,6 \pm 6,2$ & $142,8 \pm 6,5$ & $137,7 \pm 6,6$ & $138,6 \pm 7,9$ & ns \\
\hline $\mathrm{K}+(\mathrm{mEq} / \mathrm{L})$ & $3,8 \pm 0,5$ & $4,1 \pm 0,4$ & $3,7 \pm 0,5$ & $4,5 \pm 0,7^{a, c}$ & $\begin{array}{l}\mathrm{p}^{\mathrm{a}}=0,02 \\
\mathrm{p}^{\mathrm{c}}=0,03\end{array}$ \\
\hline URE (mg/dL) & $22,5 \pm 5,5$ & $22,0 \pm 7,6$ & $23,9 \pm 9,5$ & $26,1 \pm 10,3$ & ns \\
\hline CRE (mg/dL) & $1,1 \pm 0,3$ & $1,1 \pm 0,2$ & $0,9 \pm 0,3^{b 1}$ & $0,8 \pm 0,3 \mathrm{a}, \mathrm{b} 2$ & $\begin{aligned} p^{a} & =0,03 \\
p^{b 1} & =0,03 \\
p^{b 2} & =0,003\end{aligned}$ \\
\hline Alb U/Cr (mg/g) & $5,5 \pm 2,9$ & $9,1 \pm 7,9$ & $5,1 \pm 2,5$ & $8,8 \pm 6,6$ & ns \\
\hline
\end{tabular}

Caracterização quanto ao $\mathrm{n}$ (tamanho), $\mathrm{m}$ (número de mulheres) e de $\mathrm{h}$ (número de homens), idade e IMC (índice de massa corporal), sendo os valores dos dois últimos parâmetros expressos como média e desvio padrão. Os dados bioquímicos estão representados pelas médias e pelos desvios padrão dos valores de GLI (glicemia de jejum), GHB (glico-hemoglobina), GLI PP (glicemia pós-prandial), CT (colesterol total), HDLc (colesterol presente na lipoproteína de alta densidade), LDLc (colesterol presente na lipoproteína de baixa densidade), TGL (triglicérides), ALT (alanina aminotransferase), AST (aspartato aminotransferase), FAL (fosfatase alcalina), Na+ (sódio), K+ (potássio), URE (uréia), CRE (creatinina) e Alb $\mathrm{U} / \mathrm{Cr}$ (relação albumina/creatinina urinária) para os grupos Controle, HAS (com hipertensão), DM2 (com DM tipo 2) e DM2+HAS (com DM tipo 2 e hipertensão). As diferenças significativas entre os grupos estão representadas pelas letras: a. vs Controle, b. vs HAS e c. vs DM2 
A Figura 1 ilustra os resultados obtidos para identificação da mutação no gene do fator V (G1691A - fator V Leiden), e a Figura 2 ilustra os resultados obtidos para identificação da mutação no gene do fator II (G20210A), através da técnica de PCR-RFLP, em participantes deste estudo.

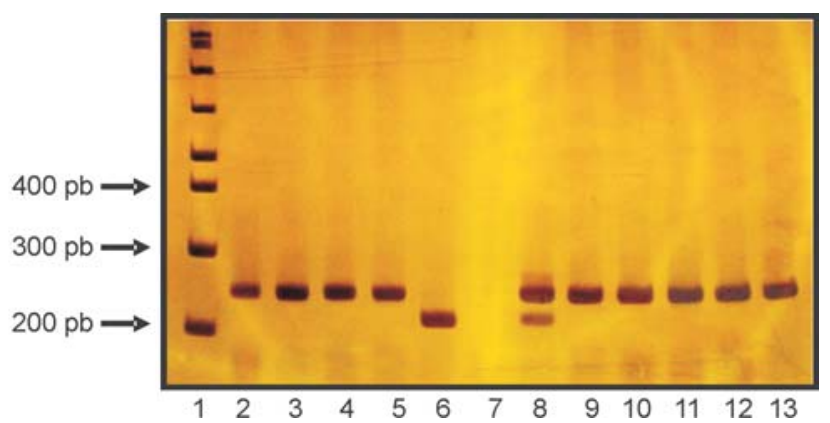

Figura 1. Identificação da mutação no gene do fator V (G1691A fator $\mathrm{V}$ Leiden)

Na canaleta 1, padrão de peso molecular; na canaleta 6 , banda de 209pb (controle homozigoto para a mutação); na canaleta 7 , branco da reação; na canaleta 8 , uma banda de 241 e outra de $209 \mathrm{pb}$ (controle heterozigoto para a mutação); e nas canaletas $2,3,4,5,9,10,11,12$ e 13 , banda de $241 \mathrm{pb}$ (indivíduos não portadores da mutação).

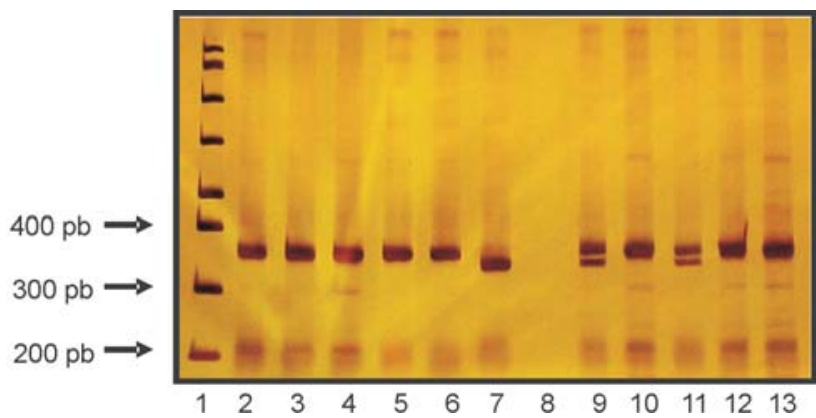

Figura 2. Identificação da mutação no gene do fator II (G20210A)

Na canaleta 1, padrão de peso molecular; na canaleta 7 , banda de 322pb (controle homozigoto para a mutação); na canaleta 8 , branco da reação; na canaleta 9 , uma banda de 345 e outra de $322 \mathrm{pb}$ (controle heterozigoto para a mutação); nas canaletas 2, 3, 4, 5, 6, 10, 12 e 13, banda de 345pb (indivíduos não portadores da mutação); e na canaleta 11, uma banda de 345 e outra de $322 \mathrm{pb}$ (indivíduo portador da mutação em heterozigose).

Os resultados dos testes moleculares obtidos neste estudo estão relacionados na Tabela 2. Os dados estão apresentados como o número de indivíduos portadores das mutações nos genes do fator V (G1691A - fator V Leiden) e do fator II (G20210A), em heterozigose nos grupos Controle, com hipertensão (HAS), com DM2 (DM 2), com DM2 e hipertensão (DM2+HAS) e o total. A freqüência de cada mutação observada para o total de indivíduos avaliados está apresentada entre parênteses. Entre os indivíduos estudados não foi identificado qualquer portador das mutações nos genes do fator V (G1691A - fator V Leiden) e do fator II (G20210A) em homozigose.

Tabela 2

Grupos Controle, HAS (com hipertensão), DM2 (com DM2), DM2+HAS (com DM2 e hipertensão) e total Os números entre parênteses indicam a freqüência observada

\begin{tabular}{ccc}
\hline & $\begin{array}{c}\text { fator V (G1691A) } \\
\text { heterozigose }\end{array}$ & $\begin{array}{c}\text { fator II (G20210A) } \\
\text { heterozigose }\end{array}$ \\
\hline Controle $(n=16)$ & 0 & 0 \\
HAS $(n=12)$ & 1 & 1 \\
DM2 $(n=7)$ & 0 & 0 \\
DM2+HAS $(n=18)$ & 0 & 1 \\
\hline Total $(n=53)$ & $1(1,9 \%)$ & $2(3,8 \%)$
\end{tabular}

\section{Discussão}

Entre os 53 indivíduos que compuseram os grupos Controle, HAS, DM2 e DM2+HAS (Tabela 2), a presença da mutação no gene do fator II (G20210A) em heterozigose foi observada em dois indivíduos: um do grupo HAS e outro do grupo DM2+HAS. A mutação no gene do fator V (G1691A) em heterozigose foi observada em um indivíduo pertencente ao grupo HAS. Considerando todos os indivíduos avaliados no estudo ( $\mathrm{n}=53)$, as freqüências observadas foram: $3,8 \%$ para a mutação no gene do fator II (G20210A) e 1,9\% para a mutação no gene do fator V (G1691A) em heterozigose.

Em São Paulo, Arruda et $\mathrm{a}^{12}$ encontraram uma prevalência de $0,7 \%{ }^{12}$ da mutação G20210A no gene do fator II em brasileiros descendentes de caucasianos e para a mutação A1691G no gene do fator V, $2 \% .{ }^{13}$ Guimarães ${ }^{14}$ encontrou uma freqüência de $6,9 \%$ da mutação no gene do fator II e de $1,7 \%$ da mutação no gene do fator $\mathrm{V}$ em mulheres de Minas Gerais. A freqüência encontrada no presente estudo muito se assemelha à encontrada por Guimarães, indicando que, possivelmente, em Minas Gerais, a incidência da mutação no gene do fator II é mais elevada.

No entanto, como as freqüências encontradas nos grupos estudados foram baixas e similares àquelas observadas na população brasileira em geral, não foi possível estabelecer correlação entre a presença da mutação e as características específicas de cada grupo, por exemplo, hipertensão e DM.

Smiles et $\mathrm{a}^{15}$ relataram a não associação da mutação G20210A com a incidência de doença cardiovascular. Estudos envolvendo pacientes diabéticos e a presença dessas mutações são muito poucos. Gardemann et al ${ }^{16}$ observaram que as mutações G20210A e G1691A não são fatores de risco associados à doença arterial coronariana. 
Krekora et $\mathrm{al}^{17}$ encontraram uma maior prevalência de fator V Leiden em pacientes com DM2 (8,2\%). Já Maser et a ${ }^{18}$ observaram uma baixa prevalência $(3,1 \%)$ e uma baixa associação entre fator $\mathrm{V}$ Leiden e doença coronariana em pacientes diabéticos, considerando que a prevalência da mutação na população branca americana é de $5,3 \% .^{19}$

Cumpre ressaltar que, no presente estudo, nenhum paciente diabético apresentou a mutação no gene do fator $\mathrm{V}$.

Assim, ainda não está claro se há ou não uma maior prevalência de fator $\mathrm{V}$ Leiden em indivíduos diabéticos e se a presença da mutação seria o maior fator de risco para o desenvolvimento de trombose nesses indivíduos. Para elucidar essa questão são necessários estudos envolvendo um maior número de pacientes.

\begin{abstract}
Because of the high prevalence of type 2 diabetes mellitus (DM2) worldwide and the high mortality rate due to thrombotic events, it is extremely important to know about changes in the hemostatic system of such patients. Factor V mutation (G1691A - factor V Leiden) in either heterozygosis or homozygosis confers the activated protein $C$ resistant phenotype, which increases the risk of thrombotic events by a factor of seven. The G20210A mutation of the prothrombin gene results in hyperprothrombinemia, increasing the risk of thrombotic events by a multiple of three. These mutations are of great relevance considering that the presence of one or both can contribute to a hypercoagulability state accelerating complications in diabetes. The aim of this study was to evaluate the incidence of these mutations in controls $(n=16), D M 2$ subjects $(n=7)$, hypertensive subjects (HAS) $(n=12)$ and DM2+HAS subjects $(n=18)$. The frequencies found were low and similar to those observed in the Brazilian population in general. It was not possible to establish any correlation among mutations and specific features of each group. It is still not clear if there is or not a higher prevalence of these mutations in diabetic individuals or if the mutation contributes to an increase in the risk of thrombotic events in these individuals. Further studies involving a larger number of patients are necessary in order to answer these questions. Rev. bras. hematol. hemoter. 2005; 27 (3):192-196.
\end{abstract}

Key words: Type 2 diabetes; factor V Leiden; prothrombin gene mutation.

\section{Agradecimentos}

Nossos agradecimentos ao CNPq/Fapemig, pelo suporte financeiro.

\section{Referências Bibliográficas}

1. Yamada T, Sato A, Nishimori T et al. Importance of hypercoagulability over hyperglycemia for vascular complication in type 2 diabetes. Diabetes Res Clin Pract 2000;49:23-31.

2. Carr ME. Diabetes mellitus: a hypercoagulable state. J Diabetes Complications 2001;15:44-54.

3. WHO - World Health Organization. Diabetes mellitus. Fact sheets, n. 138, 2002. Disponível em: http://www.who.int/inf-fs/en/fact138.html.
4. Dodson PM. Hypertension and diabetes. Curr Med Res Opin 2002; 18:48-57.

5. Bertina RM, Koeleman BPC, Koster T et al. Mutation in blood coagulation factor $\mathrm{V}$ associated with resistance to activated protein C. Nature 1994;369:64-67.

6. Dahlbäck B. Blood coagulation. Lancet 2000;355:1.627-1.632.

7. Poort P, Rosendaal FR, Reitsma PH et al. A common genetic variation in the 3 '-untranlated region of the prothrombin gene is associated with elevated plasma prothrombin levels and an increase venous thrombosis. Blood 1996;88:3.668-3.670.

8. WHO - World Health Organization. Department of Noncommunicable Disease Surveillance. Definition, diagnosis and classification of Diabetes mellitus and its complications. Geneva, 1999:59.

9. Chobanian AV, Bakris GL, Black HR et al. National High Blood Pressure Education Program Coordinating Committee. The seventh report of the Joint National Committee on prevention, detection, evaluation, and treatment of high blood pressure: The JNC 7 report. JAMA 2003;289:2.560-2.572.

10. Hirano T, Ookubo K, Kashiwazaki K et al. Vascular endothelial markers, von willebrand factor and thrombomodulin index, are specifically elevated in type 2 diabetic patients with nephropathy: comparison of primary renal disease. Clin Chim Acta 2000;2 99:65-75.

11. Dufour R, Lott JA, Nolte FS et al. Diagnosis and monitoring of hepatic injury. II. Recommendations for use of laboratory tests in screening, diagnosis, and monitoring. Clin Chem 2000; 46:2.050-2.068.

12. Arruda VR, Annichino-Bizzacchi JM, Gonçalves MS et al. Prevalence of the prothrombin gene variant (nt20210A) in venous thrombosis and arterial disease. Thromb Haemost 1997;78:1.430-1.433.

13. Arruda VR, Annichino-Bizzacchi JM, Costa FF et al. Factor V Leiden (FVQ 506) is common in a Brazilian population. Am J Hematol 1995;49:242-243.

14. Guimarães DAM. Avaliação da hemostasia, do perfil lipídico e de fatores genéticos predisponentes à trombofilia em mulheres em terapia de reposição hormonal (TRH). [Dissertação de Mestrado Programa de Pós-Graduação em Ciências Farmacêuticas]. Belo Horizonte (MG): Universidade Federal de Minas Gerais; 2003.

15. Smiles AM, Jenny NS, Tang $Z$ et al. No association of plasma prothrombin concentration or the G20210A mutation with incident cardiovascular disease: results from the Cardiovascular Health Study. Thromb Haemost 2002;87:614-621.

16. Gardemann A, Arsic T, Katz N et al. The factor II G20210A and factor V G1691A gene transitions and coronary heart disease. Thromb Haemost 1999;81:208-213.

17. Krekora K, De Lucia D, Capani F et al. Association of coagulation factor VArg 506Gln mutation with non-insulin-dependent diabetes mellitus. Lancet 1996;348:1.666-1.667.

18. Maser RE, Miele ME, Lenhard MJ et al. Lack of association of factor V Leiden and coronary heart disease in individuals with and without diabetes. Diabetes Care 1998;21:198-199.

19. Ridker PM, Miletich JP, Hennekens $\mathrm{CH}$ et al. Ethnic distribution of factor V Leiden in 4047 men and women. JAMA 1997;277: $1.305-1.307$

Avaliação: Editor e dois revisores externos.

Conflito de interesse: não declarado

Recebido: 19/07/2005

Aceito após modificações: 03/10/2005 